\title{
The effects of levels of information conflict on visual selection*
}

\section{ROBERT L. DURHAM, JUM C. NUNNALLY, and L. CHARLES LEMOND* Vanderbilt University, Nashville, Tennessee 37203}

Previous studies have reported an increasing monotonic relationship between stimulus incongruity and visual selection. The present investigation extended the former research to show the generality of this relationship by (1) using sets of stimuli with five increasing levels of novelty, (2) generating stimuli which have decreasing probabilities of occurring in the real world, and (3) extending the types of stimuli to include two new types of novelty-functional and role. The results support a theory of visual selection concerning the resolution of informational conflicts rather than a theory concerning optimal levels of arousal.

In recent years, visual exploratory behavior has emerged as an important aspect of contemporary theories of learning and motivation. This paper will be concerned with one important aspect of visual exploratory behavior, namely the tendency for $S s$ to look longer at some stimuli than at others. A measure that has been used for this purpose is called fixation dominance (FD), which refers to Ss' tendency to fixate on one part of a visual display longer than on other parts (Faw \& Nunnally, 1967). For example, eye fixations have been recorded photographically while Ss viewed pairs of pictures that differed in terms of pleasantness, complexity, familiarity, or various types of novelty (e.g., Faw \& Nunnally, 1970).

A construct of particular interest in visual exploratory behavior is information conflict. Information conflict is present whenever there are strong competing cues for identifying, naming, or otherwise attributing meaning to a stimulus. For example, a horse with a man's head may be said to contrin information conflict because Ss have difficulty in identifying the stimulus as either a horse or a man.

It has been shown in numerous studies that an incongruous stimulus will be viewed longer than its banal counterpart (Berlyne, 1958; Faw \& Nunnally, 1967; Faw \& Nunnally, 1968a). Recently, Nunnally and his colleagues have demonstrated the generality of these findings over a wide variety of stimulus conditions (e.g., Nunnally, Faw, \& Bashford, 1969). They have repeatedly found that there is an increasing monotonic relationship

-This study was supported, in part, by a research grant trom the National Institute of Child Health and Human Development, No. HDO3083, to the second author. While this research was in progress, the fint and third authors were receiving support from United States Public Henith Service tralneeships. The authors wish to express thel thanks to Mx. William $\mathrm{H}$. Wileon and Mra. Connie Lee for thelx asdetance in this experiment. between incongruity and fixation dominance. For example, when a normal Holstein cow is paired with a cow with a giraffe's neck and an airplane's tail, Ss will spend more time viewing the latter than the former.

Although the theoretical position taken by Nunnally and his colleagues predicts a monotonic relationship between levels of information conflict and amount of looking time, other theoretical positions would either flatly predict, or at least lend credence to, obtaining an inverted U-shaped relationship between the aforementioned two variables. Such theoretical positions are advanced by Berlyne (1958), Dember and Earl (1957), Fiske and Maddi (1961), and others. The reason that these and kindred theories would lead to the prediction of the inverted U-shaped relationship is that they all hinge, either directly or indirectly, on concepts concerning optimal states of arousal. It would be expected from the contents of these theoretical positions that maximum looking time would be at stimuli with intermediate levels of information conflict, and that amount of looking time would drop off on either side of that point to form the inverted U-shaped curve. In a number of investigations, Nunnally and his colleagues have provided strong evidence to indicate that the relationship between levels of information conflict and amount of looking time is monotonically increasing (Faw \& Nunnally, 1967, 1968a, b; and Nunnally, Faw \& Bashford, 1969). The purpose of the present investigation was to extend the aforementioned investigations by Nunnally and his colleagues in two important ways and a number of minor ways.

The first innovation in the present investigation was to employ new stimuli which were within the realm of probable occurrence. With the stimuli used previously, even the lowest level of novelty (Point 2 on the continuum) frequently consisted of an almost impossible object (e.g., a man with abnormally extended arms, a spotted cow, a bird with dog's ears, etc.). One of the major purposes of this study was to extend the range of stimulus materials from the banal up through the improbable but still possible to the nearly impossible kinds of pictures that had been used to depict novelty in the past. The stimuli employed in this study contained only one entirely unrealistic member (e.g., a flower growing out of a man's head, a bicycle pulling a truck van, and a cowboy with wings and propeller nose, etc.). On studying the sets of stimuli presented below, it can be seen that each item has a decreasing probability of being encountered by a $S$ in his daily life, with the fifth item being virtually impossible.

Secondly, the categories of information conflict were expanded to include two new types of conflict situations. One could rightly criticize the investigations by Nunnally and his colleagues on information conflict because they have heretofore been restricted to one means of depicting incongruity, namely to combine incongruous structural elements (e.g., a man with a dog's head, a cow with an airplane tail, a bird with hair, etc.). In those investigations, only the structure of the stimuli was manipulated; this study manipulates not only structure, but also what is defined below as functional conflict and role conflict.

Functional conflict here refers to objects which are used for some purpose other than their normal usage, e.g., a coffeepot serving as a flower vase. Role conflict refers to a person engaged in some behavior not compatible with his apparent role, e.g., a cowboy riding a bicycle. These categories will become apparent to the reader in the descriptions of the stimuli listed below.

Finally, the present investigation represents an extension of previous studies by Nunnally and his colleagues in that the stimuli were categorized on 5-point continua as opposed to the 3 and 4-point continua employed previously. The continua began with the least incongruous picture at the lowest level (first) and ranged to the most incongruous or novel picture at the highest level (fifth). Stimuli Nos. 1.4 in each set were within the realm of possible occurrence, while the fifth stimulus was virtually impossible.

\section{Subjects}

\section{METHOD}

The Ss were 40 male undergraduates enrolled in introductory psychology classes at Vanderbilt University. 


\section{Apparatus}

The $\mathrm{S}$ sat in front of a viewing box, which was 24 in. long and 21 in. high. At one end was a $21 \times 14$ in. screen. The sides of the box tapered in from the screen for $10 \mathrm{~d} / 2 \mathrm{in}$. until the width of the box was 14 in. From that point, the sides ran parallel to one another for the remaining $13 \frac{1}{2}$ in. The box was adjusted so that $S$ could rest his face in a goggle-like opening in the front and view the white plastic screen at the far end. This opening was in line with the midpoint of the screen. A chinrest was used to reduce the S's head movements. Stimulus slides were projected onto the screen from behind the viewing box by a Kodak Carousel slide projector. The projector was connected to a timing device which controlled the length of presentation of each slide.

A Bolex 16-mm movie camera with a $100-\mathrm{mm}$ lens was mounted on the side of the box and was focused on a small mirror mounted on the inside. The mirror reflected the image of the S's left eye into the lens. The eye was photographed, using Kodak high-speed infrared film running at 2 frames/sec. Except for the viewing screen and a $40-\mathrm{W}$ red light bulb in the bottom of the box, the experimental room was dark. To reduce the possibility of a S's responding to an external stimulation, each $\mathbf{S}$ wore a set of earphones which virtually eliminated any external noise.

\section{Stimuli}

Stimuli consisted of pairs of line drawings photographed on 35-mm slides and projected on the viewing screen. The inside edges of the pictures were separated horizontally by $10 \mathrm{in}$. or more. The projected image of the slide measured 21 in. wide and 14 in. high. The distance between the midpoints of the two stimuli formed an arc of $35 \mathrm{deg} 20 \mathrm{~min}$, as measured from the center of the goggle-like opening. This forced the $\mathbf{S}$ to make gross eye movements in order to bring the stimuli into his direct line of sight. Three categories of stimuli were used, with two sets of drawings in each category. Five sets contained five pictures each, while the sixth set contained only four levels of stimuli. Prior to the experiment, ratings and rankings of all sets of stimuli were made on incongruity. In addition, similar ratings and rankings were made on pleasantness, which is a potentially confounding variable. A result of these preexperimental measures indicated that the cowboy set would not separate on a clear 5-point continuum on novelty. Consequently, one member of this set was eliminated, resulting in a four-member set.

The categories of the stimuli were: functional conflict, structural conflict, and role conflict. Each conflict category contained two sets of stimulus materials. The two sets for structural conflict were a house set and a car set; the sets for functional conflict were a flower set and a trailer set; and the two sets for role conflict were a football player set and a cowboy set. All possible pairs within each set were photographed as stimulus slides. This resulted in two groups of 56 slides each. The groups were identical except for the left-right position of the more novel stimulus. Each $S$ was shown one of four random presentation orders, in which the left-right dominance was assigned randomly.

Structural conflict. The two sets of drawings in this category were the house set and the car set. In the list presented below, the number in parentheses following the stimuli in each set is the mean rank obtained from the $40 \mathrm{Ss}$ in the experiment. Each set was ranked separately, and a low-ranked number corresponds to a low ranking in novelty, while a high-ranked number corresponds to a high ranking in novelty.

Set 1-The house set contained five drawings: a normal front view of a normal house, with a door, two windows, and a chimney (1.00); the same house with disproportionate windows (2.63); the house with normal windows and a round door (2.70); the house with a Volkswagon on the roof in place of the chimney (3.88); the house with a large rabbit's head, smoke coming out of its ears in place of the chimney (4.80).

Set 2-Five drawings made up the car set: a normal side view of a car (1.03); the car missing a front wheel, leaning conspicuously on the rim (2.50); the car with its back portion replaced by a truck van (2.63); the car with a sail (4.23); and the car with a smoking brick chimney (4.63).

Functional conflict. The two sets in this category consisted of the flower set and the trailer set.

Set 3-The drawings in the trailer set were: a truck cab pulling a large trailer (1.05); a car pulling the trailer (2.00); a Volkswagon pulling the trailer (2.95); a motorcycle pulling the trailer (4.03); and finally, a bicycle pulling the trailer (4.98).

Set 4-Making up the flower set were: a single flower in a vase $(1.02)$; a flower in a coffeepot (2.65); a flower in a fishbowl with a fish swimming in the bowl $(2.85)$; a flower seemingly growing out of a hat (3.70); and a flower growing from the top of a man's head (4.75).

Role conflict. This category was composed of the football player set and the cowboy set.

Set 5-The five drawings that made up the football player set were: a football player in uniform throwing a football (1.03); the football player reading a book while in uniform (2.45); the football player pole-vaulting (2.73); the football player dressed in a jersey and helmet, but wearing a ballet skirt and one ballet shoe (3.98); the football player with a rabbit's head and tail, throwing a football (4.83).

Set 6-In contrast to the other sets which all contained five drawings, the cowboy set contained only four drawings (because only four levels could be differentiated in our preexperimental ratings). These drawings were: a cowboy riding a horse (1.08); the cowboy riding a bicycle $(1.95)$; the cowboy riding a tiger (3.05); and the cowboy with wings and a propeller nose (3.93).

The mean ranks of levels collapsed across sets were: $1.03,2.36,2.82$, 4.03 , and 4.72 .

On reading the descriptions of the pictures, it should be intuitively obvious that the highest ranked stimulus in each set concerns an essentially impossible stimulus, which would never be encountered in reality. In terms of probable occurrence of objects, the first four members (in the cowboy set, the first three members) are all within the realm of the possible. The final member in each set has essentially a zero probability of occurrence.

\section{Procedure}

The Ss were tested in individual sessions lasting approximately $\mathbf{4 5} \mathrm{min}$. Ss were escorted into the experimental room and familiarized with the viewing box. They were told that they would be shown a series of slides on the screen in back of the box. They were given a set of instructions which were intended to disguise the measurement of eye fixations, and they were informed that the study dealt with investigating pupillary dilation. [Nunnally and his colleagues have argued that many experiments on visual selection were dominated by the demand characteristics to look at stimuli in proportion to their pleasantness. This is because, in such research, Es tend to say, "Look at what you like to look at as long as you want to look at it." Faw and Nunnally (1967) found that somewhat different results are obtained in studies of visual selection when such a pleasantness set is given and when various disguises are employed.] The disguise set employed here is one that has been used in numerous studies by Nunnally and his colleagues. The verbatim instructions employed in this study are essentially the same as those presented by Faw and Nunnally (1967). 
Table 1

Mean Percent Looking Time

Level of Information Conflict

\begin{tabular}{lccccc} 
& 1 & 2 & 3 & 4 & 5 \\
\hline Set 1 & $\mathbf{4 2 . 4 7}$ & $\mathbf{5 2 . 6 3}$ & $\mathbf{4 7 . 8 5}$ & $\mathbf{5 0 . 0 9}$ & $\mathbf{5 6 . 9 6}$ \\
Set 2 & $\mathbf{4 5 . 0 1}$ & $\mathbf{4 9 . 7 2}$ & $\mathbf{5 1 . 8 6}$ & $\mathbf{5 2 . 1 0}$ & $\mathbf{5 1 . 3 1}$ \\
Set 3 & $\mathbf{4 8 . 6 4}$ & $\mathbf{4 8 . 7 0}$ & $\mathbf{5 1 . 1 3}$ & $\mathbf{5 3 . 6 3}$ & $\mathbf{4 8 . 0 8}$ \\
Set 4 & 42.27 & 49.58 & $\mathbf{5 1 . 8 2}$ & $\mathbf{5 2 . 7 6}$ & $\mathbf{5 3 . 4 6}$ \\
Set 5 & 49.61 & $\mathbf{4 4 . 9 6}$ & $\mathbf{4 7 . 2 5}$ & $\mathbf{5 2 . 4 0}$ & $\mathbf{5 5 . 7 7}$ \\
All Sets & $\mathbf{4 5 . 8 4}$ & $\mathbf{4 9 . 0 5}$ & $\mathbf{4 9 . 8 1}$ & $\mathbf{5 2 . 7 1}$ & $\mathbf{5 3 . 1 1}$ \\
Set 6 & $4 \mathbf{5 7}$ & $\mathbf{4 8 . 7 1}$ & $\mathbf{4 8 . 9 6}$ & $\mathbf{5 5 . 2 6}$ & \\
\hline
\end{tabular}

After the instructions were given, $\mathbf{S}$ was presented the 56 slides. To avoid fatigue, $S$ viewed the slides in four groups of 14 slides each, with a rest period of about $1 \mathrm{~min}$ between each group. Each slide was presented for 12 sec, and each group of slides was preceded and followed by a fixation slide, consisting of a single asterisk located at the center of the screen. The fixation slide indicated the beginning and ending of each group of slides. All Ss were informed about the fixation slides and their purpose.

At the conclusion of the viewing task, each $\mathbf{S}$ was asked to rank the stimuli in each set from most novel to least novel. Then the $S$ was reshown the first and last five slides he had previously viewed in the looking box, and asked to indicate which member of each pair he had viewed the longest. If Ss were aware of their behavior in the study, they should have been able to so demonstrate their awareness.

\section{Analysis of Photography}

The individual movie frames taken of each S's left eye were analyzed by an experienced judge who was uninformed about which stimuli the $S$ had viewed. Using the corneal reflections as an index of the eye's position, the judge recorded the position as either left, right, or center. The score of an individual $S$ was the percentage of time he spent viewing the more novel stimulus of each pair. Center fixations were not used in the analysis. Employing an identical technique, an earlier study reported an interjudge agreement of approximately $98 \%$ over a sample of 4,000 frames (Faw \& Nunnally, 1967).

\section{RESULTS}

The mean percentage scores for each level of each set are presented in Table 1.

The resulting data is best summarized by a straightforward Pearson product-moment correlation between levels of novelty and the mean rankings of the stimuli. This involved a scatter plot consisting of 25 points. The resulting correlation was $r=.73$. An inspection of the scatter plot showed no departure from linearity. The strong overall linear correlation was matched by a statistically significant linear component for the trend in each set of materials analyzed separately. In only one case (the trailer set) was the quadratic or higher level trend statistically significant.

Since the sixth set contained only four levels of information conflict, it was analyzed separately. The overall linear trend found in the previous five sets was matched in the cowboy set, $r=.88$.

In addition to the major findings presented above, each S's awareness of his performance on the visual selection task was ascertained. Each $\mathbf{S}$ was reshown the first and last five slides. A score of 1 was given if $S$ reported having viewed the more conflicting stimulus when the pair had been shown previously, and a score of 0 was given if $\mathbf{S}$ reported having viewed the less conflicting stimulus. For those stimuli, a point-biserial correlation was computed between verbal reports of viewing behavior and the percentage of time actually spent viewing the more conflicting stimulus. (Each $\mathbf{S}$ was represented 10 times in the computations.) The resulting correlation was .11.

\section{DISCUSSION}

The nearly zero correlation (.11) between actual amounts of looking time and postexperimental judgments by Ss of their behavior in the study strongly suggests that the obtained results are not due to consciously held sets of "proper" behavior for the study. If such sets had been influential while the study was in progress, Ss should have been able to reemploy those sets during the postexperimental interview.

The results of the experiment provide one suggestion regarding the difficulty of controlling extraneous factors when atcempting to manipulate an independent variable in the study of visual selection. Such an error of stimulus control occurred in the trailer set, which produced the only significant departure from the overall monotonic increasing trend. This departure from linearity is due to $\mathrm{Ss}$ spending much less time viewing the fifth-level stimulus in that set than the previous members. The fifth-level stimulus depicted a bicycle pulling a large trailer. On the postexperimental examination of the stimuli, it was determined that the size of the bicycle was too small for Ss to identify properly or to differentiate it from the previous stimulus (the motorcycle pulling a trailer). Another such error of stimulus control may be seen in the football player set, where the first-level stimulus, the football player throwing a football, was viewed longer than the second-level stimulus. This is seen as due to inadvertent conflict within that particular slide, as $\mathrm{Ss}$ reported after the study that the number on the player's uniform (01) was an improbable number for a quarterback to wear. Although there are other similar perturbances within individual sets of materials, the overall monotonic increasing trend across sets is apparent.

In addition to the aforementioned and other incidental findings, the major outcome of this study was the extension of those results previously obtained by Nunnally et al regarding the monotonic relationship between levels of novelty and amounts of looking time. As was mentioned in the introductory section of this article, the shape of this relationship has implications regarding theories of visual exploratory behavior. In the present investigation the generality of the monotonic relationship was extended to (1) more levels of novelty than had been included in any previous investigation, and (2) two types of incongruity functional and role) that had not been explicitly included in previous studies.

The obvious criticism of the aforementioned findings is that there may yet be degrees of stimulus incongruity that would result in a nonmonotonic relationship with looking time. The authors have failed in numerous attempts to construct incongruous stimuli that would be rated by either adults or children as clearly more novel than the most incongruous stimuli in the present sets. Also, it defies the imagination to think of stimuli that would be rated as clearly lore novel than, e.g., a house with a large rabbit's head, with smoke coming out of its ears in place of the chimney.

Because of some confusions that typically surround experiments like the study reported in this paper, two points should be emphasized. First, the independent variable in this experiment concerned degrees of incongruity, in the sense that each visual display contained strongly competing cues for attributing 
meaning. This is not the same as pleasantness or interestingness. More importantly, incongruity is not the same as amount of information, in the information-theoretic sense. The latter would be represented by random arrangements of dots or randomly generated polygons. Second, the dependent measure in this experiment was visual selection (amount of looking time) rather than other measures that have been applied to similar stimuli, such as memory and ratings of pleasantness. Considering the weight of the evidence from this and other studies, it should now be assumed that a monotonic increasing relationship will exist between stimulus incongruity as reflected in novelty and visual selection.

\section{REFERENCES}

BERLYNE $D$. $E$. The influence of complexity and novelty in visual floures on orienting responses. Journal of Experimental Psychology, 1958, 65, 289-296.

DEMBER, W. N., EARL, R. Analysis of exploratory, manipulatory, and curiosity behavior. Psychological Review, 1957, 64, 91-86.

FAW, T. T, \& NUNALLY, J. C. The effects on eye movements of complexity, novelty, and affective tone. Perception \& Paychophysics, 1967, 2, 263-267.

FAW, T. T., \& NUNNALLY, J. C. The influence of simulus complexity, novelty, and affective tone on childrens' visual fixations. Journal of Experimental Child Pyycholozy, 1968a, 6, 141-168.

FAW, T. T., \&UNNALLY, J. C. A new methodolozy and finding relating to visual stimulus selection in children. Psychonomic Science, 1968b, 12, 47-48.

FAW, T. T \& NUNNALLY, J. C. Eifects of familiarization with incongruous stimuli on their dominance in visual selection. Psychonomic Science, 1870, 6, 859-361.

FISKE, D. W. \& MADDI, S. R. Functions of varied experience. Homewood, III: Dorsey Press, 1961.

NUNNALLY, J. C., FAW, T. T., \& BASHFORD, M. B. The effect of degrees of incongruity on vigual selection in children and adulte. Journal of Experimental Psychology, 1969, 81, 360-364.

(Accepted for publication January 10, 1971.) 